\title{
Analysis of competitive interactions in triocultures of Drosophila melanogaster
}

\author{
Mortaza Hemmat and \\ Paul Eggleston
}

Department of Genetics and Microbiology, University of Liverpool, PO Box 147, Liverpool L69 3BX, U.K.

Competitive interactions in complex mixtures of genotypes have rarely been studied despite their obvious importance in both natural and commercial populations. Here, we describe a procedure for the analysis of competition in tripartite mixtures of Drosophila melanogaster genotypes. We have utilised a substitution design coupled with a yield-density regression analysis which describes intra- and inter-genotypic competitive effects in terms of simple linear parameters. The experimental design allows any of the competitors to be considered as the primary or indicator genotype and also incorporates variation in the relative proportions of the two associate competitors. The regression parameters are used to derive estimates of the competitive pressure exerted by each associate on the indicator genotype and also the response or sensitivity of the indicator to the competitive pressure which it faces in mixed culture. The results indicate that the joint pressure exerted by the paired associate genotypes in trioculture is equal to the sum of the individual pressures of those associates. This additive relationship holds for a variety of indicator genotypes isolated from the Texas population and appears to be a general property of Drosophila competition. We identified one indicator genotype which consistently departed from this relationship although additivity of joint pressures could be restored in combination with particular associate genotypes. The possible role of larval interference in the determination of these interactions is discussed.

\section{INTRODUCTION}

The study of competition in Drosophila has long been appreciated and has involved the establishment of a variety of experimental designs. Perhaps the most generally applied of these is the substitution or replacement design of de Wit (1960) which allows for the yield-density regression analysis of competing genotypes. Generally, series of mixed cultures are raised, throughout which one genotype is progressively replaced by another. Mather and Caligari (1981) revised this method of analysis by including the monoculture as well as the duoculture series and this led to the independent estimation of intra-genotypic and inter-genotypic competitive effects. In combination these two parameters can be used to describe how genotypes exert competitive pressure or aggression on the one hand and respond to such aggression on the other (Mather and Caligari, 1983). Hemmat and Eggleston (1988a) demonstrated that the aggression and response components of competition are separately adjustable by selection although they are not determined by independent arrays of genes. These and related studies, which have been based on the analysis of mono- and duocultures, assume an additive relationship between the aggression and response components. Although interaction of these components is rare in Drosophila competition experiments, a few examples have been recorded and these have generally been interpreted as the result of larval interference (Caligari and Mather, 1988). Competitive interactions in $D$. melanogaster mixtures with more than two genotypes have rarely been studied, despite the importance of complex mixtures in both natural and commercial populations. Calgari and Mather (1984) investigated the competition among Drosophila larvae raised in trioculture in experiments which involved a primary or indicator genotype and two associate genotypes. Both indicator and associate genotype densities were allowed to vary, but in all cases equal numbers of the two associates were used. The results of these experiments suggested that the joint pressure or aggression exerted by the two associates in trioculture was the same as the sum of their individual aggressions in duoculture. That is, aggression 
behaved in a simple additive manner. This additive relationship was further improved when the relative proportions of the two associate genotypes surviving in trioculture were taken into account, rather than the initial (equal) proportions. That is, when competition between the paired associates themselves was excluded. However, this assumed additive relationship was based on the study of only three genotypes and the experimental design allowed only one or other of the associate genotypes to be considered as the indicator. The present investigation utilised a modified experimental design in which not only the total associate genotype density was varied, but also the relative proportions of the paired associates. In this way, any of the genotypes involved in the experiment could be considered as the indicator and thus provide a thorough test of the hypothesis of additivity.

\section{EXPERIMENTAL DETAILS}

The genotypes of $D$. melanogaster used in this experiment were three inbred lines (T1, T19, T25), originating from the Texas population of $D$. melanogaster. The origin of this material has been described by Linney et al., (1971) and for all genetical characters so far investigated these inbred lines display a range of variation comparable to that found in the genetically segregating population from which they were derived. In addition, a series of long inbred strains created by the introduction of the body colour marker $\left(y^{2}\right)$ and the whiteapricot eye colour allele $\left(w^{\mathrm{a}}\right)$ to the same wild type strains (as described by Mather and Caligari, 1983) were used. The resulting strains were denoted by the prefixes $y^{2}\left(y^{2} \mathrm{~T} 1, y^{2} \mathrm{~T} 19\right.$ and $\left.y^{2} \mathrm{~T} 25\right)$ and $w^{a}$ $\left(w^{\mathrm{a}} T 1, w^{\mathrm{a}} \mathrm{T} 19\right.$ and $\left.w^{\mathrm{a}} \mathrm{T} 25\right)$. The use of three different phenotypes makes it possible to distinguish the indicator and associate competitors in duoculture and trioculture. Although the $y^{2}$ allele has been shown to have a negligible effect on the outcome of larval competition, the $w^{\mathrm{a}}$ mutation does have a detrimental effect, possibly associated with a lowering of developmental rate (Eggleston, 1987). However, it should be noted that no equivalence of the variant genotypes, for example $\mathrm{T} 1, y^{2} \mathrm{~T} 1$ and $w^{\mathrm{a}} \mathrm{T} 1$ is assumed or indeed, necessary, in these experiments.

Each competition experiment consisted of the following density series, where the figures represent the number of eggs seeded into the monoduo- and trioculture competition tubes. For all mixtures, the indicator genotype is denoted $\mathrm{A}$ and the associate genotypes B and C. In triocultures, the fixed density associate is given first and the variable density associate second.

\begin{tabular}{lllrrrrr}
\hline Indicator & A & Monoculture & 120 & 90 & 60 & 30 & 0 \\
\hline Associate & B or C & Duocultures & 0 & 30 & 60 & 90 & 120 \\
Associates & B Dr $_{1}$ & Trioculture & 0 & 30 & 30 & 30 & 30 \\
& C & & 0 & 0 & 30 & 60 & 90 \\
\hline
\end{tabular}

Whereas the design of the mono- and duoculture series is as described previously (Hemmat and Eggleston, 1988a) the trioculture design requires comment. Essentially, two independent triocultures are raised for each indicator and pair of associates. In each of these triocultures the density of one or other associate is kept constant and the other varied. Thus, the paired triocultures can be treated analytically as duocultures although together they provide all of the information necessary within a tripartite competition experiment.

For each indicator genotype, one monoculture series, six duoculture series and eighteen trioculture series were raised simultaneously. These represent all distinguishable combinations of the nine initial indicator genotypes. Consequently, the total number of cultures raised for each indicator genotype was twenty-five and for the entire experiment one hundred and eighty-nine (nine monoculture series, eighteen duoculture series and one hundred and sixty-two trioculture series).

Each vial contained $45 \mathrm{mg}$ yeast (Sigma YSC2 ), and all cultures were individually randomised and incubated at $25 \pm 0 \cdot 5^{\circ} \mathrm{C}$. Two replicate experiments were raised to provide a source of error variation. The character $P$, the proportion of eggs successfully developing into adults (Calgari, 1980) was chosen to measure competitive success. Each value of $P$ was transformed to an angle as described by Fisher and Yates (1963).

For each indicator genotype, twenty-five regression coefficients were obtained, including $b_{m}$, six $b_{d}$ and eighteen $b_{t}$ (the suffices $m, d$ and $t$ representing monoculture, duoculture and trioculture respectively). Each indicator genotype also yielded its own $e$ value representing the competitive performance of that genotype at the reference density of $N$ (120 eggs per culture). Values for the intra-genotypic competitive effect were estimated by subtracting the monoculture slope from the line of zero relationship $\left(b_{0}-b_{m}\right)$, and those for the inter-genotypic competitive effect by subtracting monoculture from duoculture or trio- 
culture slopes ( $b_{d}-b_{m}$ and $b_{t}-b_{m}$ respectively) as described by Mather and Caligari (1983) and Caligari and Mather (1984).

For each indicator genotype the validity of the linear regression model was confirmed by comparing residual regression and duplicate error variances. None of the variance ratios so obtained were significant. In addition to the competitive values and $e$-values, the mean aggression and response of each indicator genotype were estimated as well as their error variances, in duocultures and triocultures, using a diallel analysis as described by de Miranda and Eggleston, (1987).

\section{RESULTS}

The results obtained from the mono-, duo- and triocultures throughout the experiment are given in table 1. Initially, estimates for intra-genotypic competition $\left(b_{0}-b_{m}\right)$ and inter-genotypic competition in duoculture $\left(b_{d}-b_{m}\right)$ or trioculture $\left(b_{t}-\right.$ $b_{m}$ ) were obtained and used to calculate estimates of mean aggression and mean sensitivity. The former is the average competitive pressure exerted by an associate on an array of indicator genotypes and the latter is the average response of an indicator to the pressure it faces from an array of associates. The nature of the experimental design employed here is such that estimates of mean aggression and response may be obtained using either duocultures or triocultures. The triocultures, for example, were designed to behave analytically as duocultures in that the density of one of the paired associates was constant. This fixed density associate should, therefore, not affect the competitive interactions over the density series. Consider, for example, the inter-genotypic competition among three genotypes, an indicator (A) and two associates ( $B$ and $C$ ). If we remember that in the notation for triocultures, the genotype given first is maintained at constant density whereas that given second has variable density, then four estimates of inter-genotypic competition can be derived. Of these $\left(b_{d B}-b_{m}\right)$ and $\left(b_{t(C, B)}-b_{m}\right)$ should be identical, as should $\left(b_{d C}-b_{m}\right)$ and $\left(b_{t(B, C)}-b_{m}\right)$ subject, of course, to the limits of sampling variation. The use of this relationship as a test of the experimental design is feasible given the data in table 1 , but is not straightforward. A Bartlett test revealed highly significant heterogeneity among the duplicate error variances for the nine indicator genotypes $\left(\chi_{(8)}^{2}=50.06\right.$; $P<0.001)$. Clearly, an analysis of variance over all nine genotypes would not be appropriate. A more suitable procedure is to compare estimates of mean aggression and mean response obtained from duocultures with those obtained from triocultures, using a non-parametric test. The duoculture and trioculture estimates should be the same apart from variation due to sampling error. The appropriate estimates may be compiled from table 1 but are not given here for the sake of brevity. Using Spearmans test, highly significant rank correlations were found between duoculture and trioculture estimates of mean aggression $\left(r_{s}=0.92\right)$ and mean response $\left(r_{s}=0.90\right)$. Thus, as might be expected from the design of the experiment, the rank of each indicator genotype with respect to both parameters remained consistent between duocultures and triocultures. Clearly, the fixed density associate genotype does not affect the competitive interactions in trioculture and behaves as a simple constant over the density series.

A modified version of the technique of Caligari and Mather (1984) was employed in testing the additivity of aggression of the associate genotypes in trioculture. Their analysis involved the use of density series in which equal numbers of the paired associate genotypes were always used. It was proposed that the joint pressure or aggression exerted on the indicator by the paired associate genotypes in trioculture was equal to the sum of the pressures exerted individually by those associate genotypes. In the present experiment two types of trioculture exist for each pair of associates with one or other of the pair maintaining a constant density.

If joint pressure behaves additively, as proposed above, then we can write the following relationship for the experimental design employed here:

$$
\begin{aligned}
f= & {\left[\left(u C_{A, B}+v C_{A, C}\right)-C_{A(B, C)}\right] } \\
& +\left[\left(u C_{A, B}+v C_{A, C}\right)-C_{A(C, B)}\right] .
\end{aligned}
$$

Where, for example $C_{A, B}$ is the inter-genotypic competitive effect of associate $B$ on indicator $A$ in duoculture and $C_{A(B, C)}$ is the inter-genotypic competitive effect of the paired associates $B$ and $C$ on the indicator $\mathrm{A}$; $\mathrm{B}$ having constant density and $\mathrm{C}$ variable density. The coefficients $u$ and $v$ are the proportions of the associate genotypes (B and C) which emerge following competitive interaction in both duoculture and trioculture. They therefore reflect the competition which takes place between the associate genotypes themselves and act as a more appropriate weight for the various competitive values than do the initial proportions of the associate genotypes with which the triocultures were initiated. 
Table 1 Regression coefficients estimating intra-genotypic competition $\left(b_{m}\right)$ and inter-genotypic competition in duoculture $\left(b_{d}\right)$ or trioculture $\left(b_{t}\right)$ for wild type, yellow and white-apricot indicator genotypes. In all cases the indicator genotype is denoted $\mathrm{A}$ and the associate genotypes B and/or C. The notation employed for triocultures is such that the first associate is maintained at constant density whereas the second associate has variable density (i.e., $b_{t(B, C)}$; associate $\mathrm{B}$ has fixed density, associate $\mathrm{C}$ has variable density). The remaining parameters are involved in the test of additivity of joint pressure from the paired associates. $u$ and $v$ are the relative proportions of the fixed and variable density associates obtained following competition in duoculture and trioculture. Only $u$ is given but $v$ may be obtained as $(1-u)$. The parameter $f$ is a test of additivity (see text for details) and its departure from zero is tested by $\chi_{(1)}^{2}$. Levels of significance are shown as $*, 0.05>P>0.01 ; * *, 0.01>P>0.001 ;{ }^{* * *}$, $P<0.001$

\begin{tabular}{|c|c|c|c|c|c|c|c|c|c|c|c|}
\hline A & B & $\mathrm{C}$ & $b_{m}$ & $b_{d B}$ & $b_{d C}$ & $b_{t(B, C)}$ & $b_{t(C, B)}$ & $u$ & $f$ & $V_{f}$ & $\chi_{(1)}^{2}$ \\
\hline $\mathrm{T} 1$ & $y^{2} \mathrm{~T} 1$ & $w^{\mathrm{a}} \mathrm{T} 1$ & -0.3204 & $-0 \cdot 1699$ & -0.0577 & $-0 \cdot 1019$ & -0.0876 & $0: 270$ & 0.0135 & 0.0091 & 0.0201 \\
\hline T19 & $y^{2} \mathrm{~T} 1$ & $w^{\mathrm{a}} \mathbf{T} 1$ & $-0 \cdot 1708$ & -0.0553 & -0.0164 & 0.0220 & -0.0840 & 0.216 & 0.0124 & 0.0060 & 0.0257 \\
\hline $\mathrm{T} 25$ & $y^{2} \mathrm{~T} 1$ & $w^{\mathrm{a}} \mathbf{T} 1$ & -0.0932 & -0.0839 & -0.0264 & 0.0155 & -0.0900 & 02231 & -0.0049 & 0.0040 & 0.0059 \\
\hline $\mathrm{T} 1$ & $y^{2} \mathrm{~T} 1$ & $w^{\mathrm{a}} \mathrm{T} 19$ & -0.3204 & -0.1699 & -0.1548 & -0.1474 & -0.1783 & 0.347 & 0.0056 & 0.0088 & 0.0036 \\
\hline T19 & $y^{2} \mathbf{T} 1$ & $w^{\mathrm{a}} \mathrm{T} 19$ & $-0 \cdot 1708$ & -0.0553 & -0.1122 & -0.0901 & -0.0144 & 0.359 & -0.0790 & 0.0055 & $1 \cdot 1301$ \\
\hline $\mathrm{T} 25$ & $y^{2} \mathrm{~T} 1$ & $w^{\mathrm{a}} \mathrm{T} 19$ & -0.0932 & -0.0839 & -0.0369 & -0.0249 & -0.1163 & 0.347 & 0.0348 & 0.0038 & 0.3217 \\
\hline T1 & $y^{2} \mathrm{~T} 1$ & $w^{\mathrm{a}} \mathbf{T} 25$ & -0.3204 & -0.1699 & -0.0962 & -0.1121 & $-0 \cdot 1424$ & $0 \cdot 298$ & 0.0182 & 0.0090 & 0.0369 \\
\hline T19 & $y^{2} \mathrm{~T} 1$ & $w^{\mathrm{a}} \mathrm{T} 25$ & $-0 \cdot 1708$ & -0.0553 & -0.0364 & 0.0134 & -0.0110 & 0.315 & -0.0871 & 0.0056 & $1 \cdot 3472$ \\
\hline $\mathrm{T} 25$ & $y^{2} \mathrm{~T} 1$ & $w^{\mathrm{a}} \mathrm{T} 25$ & -0.0932 & -0.0839 & -0.0514 & 0.0035 & $-0 \cdot 1033$ & $0 \cdot 324$ & -0.0241 & 0.0038 & 0.1524 \\
\hline $\mathrm{T} 1$ & $y^{2} \mathrm{~T} 19$ & $w^{\mathrm{a}} \mathbf{T} 1$ & -0.3204 & -0.1693 & -0.0577 & -0.0812 & -0.1376 & $0 \cdot 326$ & 0.0307 & 0.0088 & $0 \cdot 1069$ \\
\hline T19 & $y^{2} \mathrm{~T} 19$ & $w^{\mathrm{a}} \mathrm{T} 1$ & $-0 \cdot 1708$ & -0.0758 & -0.0164 & -0.0112 & -0.0917 & 0.295 & 0.0350 & 0.0057 & 0.2160 \\
\hline $\mathrm{T} 25$ & $y^{2} \mathrm{~T} 19$ & $w^{\mathrm{a}} \mathbf{T} 1$ & -0.0932 & -0.0416 & -0.0264 & 0.0059 & -0.0614 & 0.322 & -0.0066 & 0.0038 & 0.0114 \\
\hline $\mathrm{T} 1$ & $y^{2} \mathrm{~T} 19$ & $w^{\mathrm{a}} \mathrm{T} 19$ & $-0 \cdot 3204$ & -0.1693 & -0.1548 & -0.2223 & -0.1568 & 0.451 & 0.0564 & 0.0085 & 0.3734 \\
\hline T19 & $y^{2} \mathrm{~T} 19$ & $w^{\mathrm{a}} \mathrm{T} 19$ & -0.1708 & -0.0758 & -0.1122 & -0.0802 & -0.0666 & 0.452 & -0.0447 & 0.0054 & 0.3697 \\
\hline $\mathrm{T} 25$ & $y^{2} \mathrm{~T} 19$ & $w^{\mathrm{a}} \mathrm{T} 19$ & -0.0932 & -0.0416 & -0.0369 & -0.0374 & $-0 \cdot 1427$ & 0.466 & $0 \cdot 1019$ & 0.0036 & $2 \cdot 8436$ \\
\hline $\mathrm{T} 1$ & $y^{2} \mathrm{~T} 19$ & $w^{\mathrm{a}} \mathrm{T} 25$ & -0.3204 & -0.1693 & -0.0962 & $-0 \cdot 1016$ & -0.1819 & 0.446 & 0.0259 & 0.0085 & 0.0786 \\
\hline T19 & $y^{2} \mathrm{~T} 19$ & $w^{\mathrm{a}} \mathrm{T} 25$ & $-0 \cdot 1708$ & -0.0758 & -0.0364 & -0.0526 & -0.0687 & 0.411 & 0.0161 & 0.0054 & 0.0477 \\
\hline $\mathrm{T} 25$ & $y^{2} \mathrm{~T} 19$ & $w^{\mathrm{a}} \mathrm{T} 25$ & -0.0932 & -0.0416 & -0.0514 & -0.0254 & -0.0490 & 0.425 & -0.0201 & 0.0037 & $0 \cdot 1096$ \\
\hline $\mathrm{T} 1$ & $y^{2} \mathrm{~T} 25$ & $w^{\mathrm{a}} \mathbf{T} 1$ & -0.3204 & -0.1091 & -0.0577 & 0446 & -0.0420 & 0.506 & -0.0808 & 0.0085 & 0.7685 \\
\hline T19 & $y^{2} \mathrm{~T} 25$ & $w^{\mathrm{a}} \mathrm{T} 1$ & $-0 \cdot 1708$ & -0.0611 & -0.0164 & -0.0012 & 0.0368 & 0.504 & 0.0097 & 0.0054 & 0.0176 \\
\hline $\mathrm{T} 25$ & $y^{2} \mathbf{T} 25$ & $w^{\mathrm{a}} \mathbf{T} 1$ & -0.0932 & -0.0184 & -0.0264 & -0.0578 & -0.0443 & 0.500 & 0.0573 & 0.0036 & 0.9003 \\
\hline $\mathrm{T} 1$ & $y^{2} \mathrm{~T} 25$ & $w^{\mathrm{a}} \mathrm{T} 19$ & -0.3204 & -0.1091 & -0.1548 & -0.0216 & -0.0370 & 0.580 & -0.1980 & 0.0086 & $4.5735^{*}$ \\
\hline T19 & $y^{2} \mathrm{~T} 25$ & $w^{\mathrm{a}} \mathrm{T} 19$ & -0.1708 & 0.0611 & -0.1122 & -0.0332 & 0.0285 & 0.600 & -0.0117 & 0.0055 & 0.0252 \\
\hline $\mathrm{T} 25$ & $y^{2} \mathrm{~T} 25$ & $w^{\mathrm{a}} \mathrm{T} 19$ & -0.0932 & -0.0184 & -0.0369 & -0.0134 & 0.0293 & 0.602 & -0.0674 & 0.0037 & $1 \cdot 2294$ \\
\hline T1 & $y^{2} \mathrm{~T} 25$ & $w^{\mathrm{a}} \mathrm{T} 25$ & -0.3204 & $-0 \cdot 1091$ & -0.0962 & -0.1209 & -0 & 0.681 & -0.0305 & 39 & 0 . \\
\hline T19 & $y^{2} \mathrm{~T} 25$ & $w^{\mathrm{a}} \mathrm{T} 25$ & -0.1708 & 0.0611 & -0.0364 & -0.0038 & 0.0878 & 0.708 & -0.0187 & 0.0057 & 0.0616 \\
\hline $\mathrm{T} 25$ & $y^{2} \mathbf{T} 25$ & $w^{\mathrm{a}} \mathrm{T} 25$ & -0.0932 & -0.0184 & -0.0514 & -0.0911 & -0.0824 & $0 \cdot 728$ & $0 \cdot 1188$ & 0.0039 & 3.6157 \\
\hline$y^{2} \mathrm{~T} 1$ & $\mathrm{~T} 1$ & $w^{\mathrm{a}} \mathrm{T} 1$ & -0.0697 & 0.0381 & 0.0516 & 0.0760 & $0 \cdot 1056$ & 0.497 & -0.0918 & 0.0053 & $1 \cdot 5958$ \\
\hline$y^{2} T 19$ & $\mathrm{~T} 1$ & $w^{\mathrm{a}} \mathrm{T} 1$ & -0.0467 & 0.0614 & 0.0131 & 0.0752 & 0.0697 & 0.485 & -0.0718 & 0.0057 & 0.9090 \\
\hline$y^{<} T 25$ & $\mathrm{~T} 1$ & $w^{\mathrm{a}} \mathbf{T} 1$ & -0.2055 & 0.0244 & -0.0835 & 0.0010 & -0.0693 & 0.484 & 0.0057 & 0.0096 & 0.0039 \\
\hline$y^{2} \mathrm{~T} 1$ & T1 & $w^{\mathrm{a}} \mathrm{T} 19$ & -0.0697 & 0.03 & -0.0880 & 0.0 & $0 \cdot 1868$ & 0.609 & -0.2136 & 0.0 & $8 \cdot 5018^{* *}$ \\
\hline$y^{2} T 19$ & $\mathrm{~T} 1$ & $w^{\mathrm{a}} \mathrm{T} 19$ & -0.0467 & 0.0614 & 0.0130 & 0.0194 & $0 \cdot 1007$ & 0.608 & -0.0352 & 0.0058 & 0.2155 \\
\hline$y^{2} T 25$ & $\mathrm{~T} 1$ & $w^{\mathrm{a}} \mathrm{T} 19$ & -0.2055 & 0.0244 & -0.0850 & 0.0244 & -0.0175 & 0.597 & -0.0463 & 0.0098 & 0.2194 \\
\hline$y^{2} \mathrm{~T} 1$ & $\mathrm{~T} 1$ & $w^{\mathrm{a}} \mathrm{T} 25$ & -0.0697 & 0.0381 & -0.0234 & 0.1835 & $0 \cdot 2029$ & 0.695 & -0.3491 & 0.0055 & $21-9571^{* * *}$ \\
\hline$y^{2} T 19$ & $\mathrm{~T} 1$ & $w^{\mathrm{a}} \mathrm{T} 25$ & -0.0467 & 0.0614 & 0.0956 & 0.0542 & 0.0405 & 0.676 & 0.0503 & 0.0059 & 0.4274 \\
\hline$y^{2} T 25$ & $\mathrm{~T} 1$ & $w^{\mathrm{a}} \mathrm{T} 25$ & -0.2055 & & -0.1195 & -0.0506 & -0.0901 & 0.709 & $0 \cdot 1057$ & 0.0102 & 1.0962 \\
\hline$y^{2} \mathrm{~T} 1$ & T19 & $w^{\mathrm{a}} \mathbf{T} 1$ & -0.0697 & 0.0675 & 0.0516 & $0 \cdot 1256$ & $0 \cdot 1919$ & 0.386 & -0.2020 & 0.0054 & $7 \cdot 5933^{* *}$ \\
\hline$y^{2} \mathrm{~T} 19$ & T19 & $w^{\mathrm{a}} \mathbf{T} 1$ & -0.0467 & 0.0445 & 0.0131 & $0 \cdot 1214$ & $0 \cdot 1021$ & 0.375 & -0.1737 & 0.0058 & $5 \cdot 2095^{*}$ \\
\hline$y^{2} T 25$ & T19 & $w^{\mathrm{a}} \mathrm{T} 1$ & $-0 \cdot 2055$ & -0.0487 & -0.0835 & $-0 \cdot 1660$ & $-0 \cdot 1008$ & 0.362 & 0.1250 & 0.0099 & $1 \cdot 5805$ \\
\hline$y^{2} \mathrm{~T} 1$ & T19 & $w^{\mathrm{a}} \mathrm{T} 19$ & -0.0697 & 0.0675 & -0.0880 & 0.0894 & $0 \cdot 1540$ & 0.517 & -0.2586 & 0.0053 & $12 \cdot 6548^{* * *}$ \\
\hline$y^{2} \mathrm{~T} 19$ & T19 & $w^{\mathrm{a}} \mathrm{T} 19$ & -0.0467 & 0.0445 & 0.0130 & 0.0398 & 0.0464 & 0.542 & -0.0261 & 0.0057 & 0.1193 \\
\hline$y^{2} T 25$ & T19 & $w^{\mathrm{a}} \mathrm{T} 19$ & -0.2055 & -0.0487 & -0.0850 & $-0 \cdot 1150$ & -0.0275 & 0.515 & 0.0099 & 0.0096 & 0.0101 \\
\hline$y^{2} \mathrm{~T} 1$ & T19 & $w^{\mathrm{a}} \mathrm{T} 25$ & -0.0697 & 0.0675 & -0.0234 & $0 \cdot 1188$ & 0.0707 & 0.474 & -0.2040 & 0.0053 & $7 \cdot 8720^{* *}$ \\
\hline$y^{2} \mathrm{~T} 19$ & T19 & $w^{\mathrm{a}} \mathrm{T} 25$ & -0.0467 & 0.0445 & 0.0956 & 0.0400 & 0.0961 & 0.535 & 0.0004 & 0.0057 & 0.0000 \\
\hline$y^{2} T 25$ & T19 & $w^{\mathrm{a}} \mathrm{T} 25$ & $-0 \cdot 2055$ & -0.0487 & -0.1195 & -0.1458 & -0.0943 & 0.561 & 0.0805 & 0.0097 & 0.6695 \\
\hline$y^{2} \mathrm{~T} 1$ & $\mathrm{~T} 25$ & $w^{\mathrm{a}} \mathbf{T} 1$ & -0.0697 & $0 \cdot 1860$ & 0.0516 & $0 \cdot 1633$ & $0 \cdot 1082$ & 0.518 & -0.0292 & 0.0053 & 0.1609 \\
\hline$y^{2} \mathrm{~T} 19$ & $\mathrm{~T} 25$ & $w^{\mathrm{a}} \mathrm{T} 1$ & -0.0467 & 0.0413 & 0.0131 & 0.0493 & 0.0891 & 0.513 & -0.1253 & 0.0057 & $2 \cdot 7655$ \\
\hline$y^{2} T 25$ & $\mathrm{~T} 25$ & $w^{\mathrm{a}} \mathrm{T} 1$ & -0.2055 & -0.0240 & -0.0835 & -0.0482 & -0.0717 & 0.537 & 0.0168 & 0.0097 & 0.0292 \\
\hline
\end{tabular}


Table 1 continued

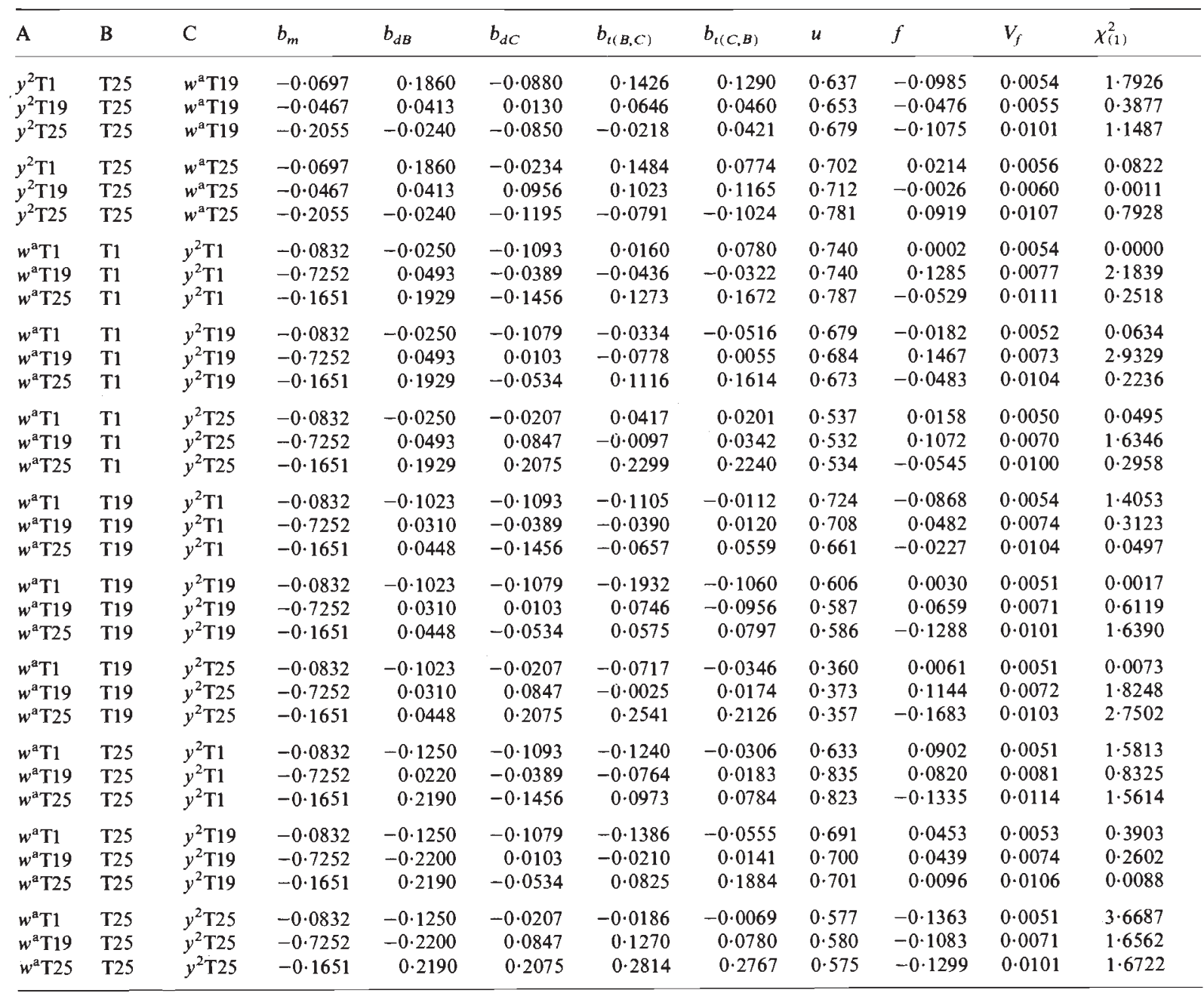

Since each $C$ value is equivalent to $\left(b_{d}-b_{m}\right)$ or $\left(b_{t}-b_{m}\right)$ and $b_{m}$ is the same for all $C$ values within each indicator genotype, equation (1) can be rewritten as:

$$
\begin{array}{r}
f=\left[\left(u b_{d B}+v b_{d C}\right)-b_{t(B, C)}\right] \\
+\left[\left(u b_{d B}+v b_{d C}\right)-b_{t(C, B)}\right]
\end{array}
$$

or simplifying:

$$
f=2 u b_{d B}+2 v b_{d C}-b_{t(B, C)}-b_{t(C, B)} .
$$

Given the hypothesis of additivity of joint pressure as described previously, we should find that $f=0$ within the limits of sampling error. In order to test this assumption, the variance of $f$ or $V_{f}$ is required.

Each competitive value in the derivation of $f$ takes the form $\left(b_{d}-b_{m}\right)$ or $\left(b_{t}-b_{m}\right)$. Although $b_{m}$ is a constant for each indicator genotype, the vari- ance of the competitive value must take into account the covariance of $b_{d}$ or $b_{t}$ with $b_{m}$.

The variances and covariances of the slopes are obtained as described by Mather and Caligari (1981). The appropriate expression therefore takes the form:

$$
V_{c}=\left(V_{b}-W_{b . b}\right) V_{E}
$$

where $V_{E}$ is the appropriate replicate error variance. Using linear functions, the variance of $f$ is derived as:

$$
\begin{aligned}
V_{f}= & u^{2}\left[\left(V_{b}-W_{b . b}\right) V_{E}\right]+v^{2}\left[\left(V_{b}-W_{b . b}\right) V_{E}\right] \\
& \left.+\left[V_{b}-W_{b . b}\right) V_{E}\right]+u^{2}\left[\left(V_{b}-W_{b . b}\right) V_{E}\right] \\
& +v^{2}\left[\left(V_{b}-W_{b . b}\right) V_{E}\right]+\left[\left(V_{b}-W_{b . b}\right) V_{E}\right] \\
= & 2\left(1+u^{2}+v^{2}\right)\left[\left(V_{b}-W_{b . b}\right) V_{E}\right] .
\end{aligned}
$$


Remembering that $(u+v)=1$ and noting that $1+$ $u^{2}+v^{2}=2(1-u v)$.

This simplifies to:

$$
V_{f}=4(1-u v)\left[\left(V_{b}-W_{b . b}\right) V_{E}\right]
$$

The deviation of $f$ from zero can then be tested quite simply as:

$$
\chi_{(1)}^{2}=f^{2} / V_{f}
$$

as described by Caligari and Mather, 1984.

\section{AN EXAMPLE}

Taking the case where T19 is the indicator genotype with $y^{2} \mathrm{~T} 25$ and $w^{\mathrm{a}} \mathrm{T} 1$ as the associates, the slopes obtained from competition in duoculture $\left(b_{d}\right)$ and

\begin{tabular}{|c|c|c|c|c|}
\hline \multirow[b]{2}{*}{ Associate } & \multicolumn{2}{|c|}{ Duoculture $\left(b_{d}\right)$} & \multicolumn{2}{|c|}{ Trioculture $\left(b_{t}\right)$} \\
\hline & $y^{2} \mathrm{~T} 25$ & $w^{\mathrm{a}} \mathrm{T} 1$ & $\begin{array}{l}y^{2} \mathrm{~T} 25, \\
w^{\mathrm{a}} \mathrm{T} 1\end{array}$ & $\begin{array}{l}w^{\mathrm{a}} \mathrm{T} 1, \\
y^{2} \mathrm{~T} 25\end{array}$ \\
\hline
\end{tabular}
trioculture $\left(b_{t}\right)$ were as follows:

Indicator

T19 $\quad 0.061139 \quad-0.0164204 \quad-0.00125377 \quad 0.0368415$

With respect to the triocultures, the first of each pair of associates is the fixed density genotype and the second has variable density. $u$ and $v$ are the proportions of the associate genotypes $y^{2} \mathrm{~T} 25$ and $w^{\mathrm{a}} \mathrm{T} 1$ respectively and are estimated from the numbers of each genotype emerging after competition in duoculture and trioculture. In this example $u$ and $v$ are estimated as 0.504 and 0.496 respectively. By substituting these values in equation (3) we obtain $f=0 \cdot 009751$.

Given that the variances and covariances of the appropriate slopes are $V_{b}=0.000096787$ and $W_{b . b}=0.000017422$ and that the duplicate error variance is $V_{E}=22.622720$, we can substitute into equation (5) to obtain $V_{f}=0.005386$.

Thus, using equation (6) we obtain $\chi_{(1)}^{2}=0 \cdot 176$; $P>0.05$.

Clearly, in this example there is no evidence to suggest that $f$ departs significantly from zero. In fact, the agreement is remarkably good.

The additivity test described above was repeated for each of the 81 tripartite mixtures involved in the experiment (table 1). It is clear that in the majority of cases there is no evidence of a failure of the assumption that $f=0$. This lends strong support to the hypothesis that the joint pressure exerted by paired associates in trioculture is equal to the sum of the individual pressures.
However, a proportion of the triocultures (seven out of 81 ) do show a significant deviation of $f$ from zero. A simple calculation would suggest that at the 5 per cent level of significance, approximately four values of $(f)$ might be found to depart significantly from zero, simply by chance. In this case we have seven significant values which would appear to exceed that expectation. However, inspection of table 1 shows that five of the seven significant $\chi^{2}$ values and, moreover, the five showing the greatest deviation of $(f)$ from zero involve $y^{2} \mathrm{~T} 1$ as the indicator genotype. The unusual behaviour of this indicator genotype is also apparent from a pooled $\chi^{2}$ analysis testing the overall departure of $(f)$ from zero for each of the nine indicator genotypes. Of the values obtained, only that for the genotype $y^{2} \mathrm{~T} 1$ as indicator is significant $\left(\chi_{(9)}^{2}=62 \cdot 21 ; P<0 \cdot 001\right)$. It appears likely, therefore, that the low significant values obtained for T1 and $y^{2}$ T19 $\left(\chi_{[1]}^{2}=4.57\right.$ and $\chi_{[1]}^{2}=$ $5 \cdot 21$ respectively, table 1$)$ may well represent chance events.

Further inspection of the behaviour of the $y^{2} \mathrm{~T} 1$ indicator genotype in trioculture shows that the significant deviation of $(f)$ from zero cannot be found in all cases. For example, none of the triocultures in which one of the paired associate genotypes was $\mathrm{T} 25$ show a significant departure of $(f)$ from zero. Neither does the trioculture in which all competitors are derived from $\mathrm{T} 1$ (i.e., T1, $y^{2} \mathrm{~T} 1$, $\left.w^{\mathrm{a}} \mathrm{T} 1\right)$. These data suggest that departure from additivity of joint pressure may be an intrinsic property of the $y^{2} \mathrm{~T} 1$ indicator genotype. These experiments, however, are unable to elucidate the underlying cause of this unusual behaviour, although a possible interpretation is discussed below.

\section{DISCUSSION}

Calgari and Mather (1984) offered a model for the analysis of genetically heterogeneous mixtures of three genotypes of $D$. melanogaster. In the experiment reported here an attempt has been made to modify this analysis into a form which is sufficiently flexible to allow any genotype to be considered as indicator. The design includes triocultures in which one of the paired associate genotypes is maintained at constant density while the other is allowed to vary. This results in the estimates of trioculture slopes $\left(b_{t}\right)$ being analytically comparable to those for duoculture slopes $\left(b_{d}\right)$. The experiments of Caligari and Mather (1984) suggested that the joint pressure exerted by 
the paired associate genotypes in trioculture was equal to the sum of the individual pressures of those associates. The results obtained in this investigation, involving triocultures over a much wider range of indicator genotypes, supported this hypothesis. It may be concluded that additivity of joint pressures exists not only for triocultures initiated with associate genotypes at equal density but also for those where associate densities are allowed to vary. The use of nine separate indicator genotypes resulted in the identification of one which showed aberrant behaviour. Out of a total of eighty-one triocultures raised, only seven did not show additivity of joint pressure and five of these involved $y^{2} \mathrm{~T} 1$ as the indicator genotype. Consequently, it was proposed that departure from additivity of joint pressure may be an intrinsic property of this genotype. However, this departure was not found for all triocultures which involved the $y^{2} \mathrm{~T} 1$ indicator. For example, those triocultures in which T25 was one of the paired associate genotypes showed no significant departure from additivity. The mechanisms underlying this phenomenon are not known but it is possible that detoxification of larval metabolic products is involved (Botella, et al., 1985; Moya, et al., 1988). Detoxification is defined as genetic variation in the ability of $D$. melanogaster genotypes to convert toxic metabolic products released by competitors to a non-toxic form. Based on this hypothesis, larval interference due to the excretion or secretion of metabolic products is a component of all competitive interactions (Hemmat and Eggleston, $1988 b$ ). The quality and quantity of these metabolic products is a property of individual genotypes, as is the ability to counteract and detoxify these products.

Although such processes could perhaps account for the unusual behaviour of the triocultures in which $y^{2} \mathrm{~T} 1$ was the indicator genotype, it would be wrong to speculate in the absence of experimental results. Clearly, some aspect of the behaviour of $y^{2} \mathrm{~T} 1$ results in a departure from additivity of joint pressure and yet this departure can be overcome by the presence of T25 as an associate. The processes of larval interference and detoxification of metabolic residues would seem to be reasonable candidates for further investigation with respect to the complementary function apparently supplied by $\mathrm{T} 25$. This hypothesis might also account for the behaviour of the $\left(y^{2} \mathrm{~T} 1: \mathrm{T} 1: w^{\mathrm{a}} \mathrm{T} 1\right)$ trioculture which also showed a restoration of additivity of joint pressure (table 1 ). Since each of these genotypes may be considered as identical, save for the incorporation of alterna- tive phenotypic markers it may be that whatever metabolic residues are produced by the associates would not be toxic to the $y^{2} \mathrm{~T} 1$ indicator. Conversely, this genotype may be able to cope with its own (or putatively identical) residues, but not those of a more different genotype.

The results of these experiments demonstrate quite clearly the additivity of joint pressure for paired associate genotypes in the vast majority of triocultures. Together with the earlier results of Caligari and Mather (1984) they suggest that such additivity may well be a general property of competition in multiple genotype cultures of $D$. melanogaster. This general property may be summarised as:

$f=\left[b_{n(B, C, D, \ldots, n)}-b_{d B}-b_{d C}-b_{d D}-\cdots b_{d n}\right]=0$

to show that the joint pressure exerted on the indicator by a mixture of $n$ genotypes is simply the sum of the individual pressures of those $n$ genotypes. It may, however, be unwise to extrapolate such a principle beyond larval competition in Drosophila without further experimental evidence. For example, results obtained so far indicate that aggression $(a)$ and response $(r)$ parameters tend to combine additively in Drosophila with only rare instances of interaction. However, the same is not true of competition in Lolium perenne (perennial ryegrass) where interaction of the parameters is much more apparent (Mather and Caligari, 1983). It would be of great interest to investigate the behaviour of joint pressures in an alternative organism such as this.

Acknowledgements We would like to thank Dr J. R. de Miranda for helpful discussion and Lynda Mitchell for technical assistance. The manuscript was prepared by Hilda Longworth to whom we express our gratitude. Financial support was provided by The Lister Institute of Preventive Medicine (Fellowship to PE) and the Iranian Ministry of Education.

\section{REFERENCES}

BOTELla, L. M., MOYA, A., GONZALEZ, C. AND menSUA, J. L. 1985. Larval stop, delayed development and survival in overcrowded cultures of Drosophila melanogaster: effect of urea and uric acid. J. Insect. Physiol. 31, 179-185.

CAligARI, P. D. S. 1980. Competitive interactions in Drosophila melanogaster. 1. Monocultures, Heredity, 45, 219-231.

CAligari, P. D. S. AND MATHER, K. 1984. Competitive interactions in Drosophila melanogaster. III. Triocultures. Heredity, 52, 255-264.

CALIGARI, P. D. S. AND MATHER, K. 1988. Competitive interactions in Drosophila melanogaster. IV. Chromosome assay. Heredity, 60, 355-366. 
DE MIRANDA, J. R. AND EGGLESTON, P. 1987. A comparison of substitution and addition designs for the analysis of competitive interactions in Drosophila melanogaster. Heredity, 58, 279-288.

DE WIT, C. T. 1960. On competition. Versl. Landbouwk. Onderz. Ned., 66, 1-82.

EGGLESTON, P. 1987. The use of genetic markers in the analysis of competitive interactions in Drosophila melanogaster. Genetica, 72, 181-186.

FISHER, R. A. AND YATES, F. 1963. Statistical tables for biological agricultural and medical research. 6th edn, Oliver and Boyd, Edinburgh.

HEMMAT, M. AND EGGLESTON, P. 1988a. Competetitive interactions in Drosophila melanogaster: recurrent selection for aggression and response. Heredity, 60, 129-137.
HEMMAT, M. AND EGgleston, P. 1988b. Competitive interactions in Drosophila melanogaster: genetic variation for interference through media conditioning. Heredity, 61, 347-354.

LINNEY, R., BARNES, B. W. AND KEARSEY, M. J. 1971. Variation for metrical characters in Drosophila populations. Heredity, 27, 163-174.

MATHER, K. AND CALIGARI, P. D. S. 1981. Competitive interactions in Drosophila melanogaster. II. Measurement of competition. Heredity, 46, 239-254.

MATHER, K. AND CAligari, P. D. S. 1983. Pressure and response in competitive interactions. Heredity, 51, 435-454.

MOYA, A., GONZALEZ, C. AND MENSUA, J. L. 1988. Larval Competition in Drosophila melanogaster: frequency dependence of viability. Theor. Appl. Genet., 75, 366-377. 